\title{
The respiratory complexes I from the mitochondria of two Pichia species
}

\author{
Hannah R. Bridges, Ljuban Grgic, Michael E. Harbour, and Judy Hirst*
}

The Medical Research Council Mitochondrial Biology Unit, Wellcome Trust / MRC Building, Hills Road, Cambridge, CB2 0XY, U. K.

*Author to whom correspondence should be addressed:

Medical Research Council Mitochondrial Biology Unit, Wellcome Trust / MRC Building, Hills Road, Cambridge, CB2 0XY, U. K.

Tel : +44 1223 252810, Fax : +44 1223 252815, E-mail : jh@mrc-mbu.cam.ac.uk

Page heading title: The complexes I from Pichia species

\section{Keywords:}

NADH:ubiquinone oxidoreductase

Complex I

Pichia pastoris

Pichia angusta

Electron transport chain

Mitochondria

\author{
Abbreviations: \\ ACMA, 9-amino-6-chloro-2-methoxyacridine; \\ DDM, n-dodecyl- $\beta$-D-maltopyranoside; \\ $\mathrm{DQ}$, decylubiquinone; \\ FCCP, carbonylcyanide-p-trifluoromethoxyphenylhydrazone; \\ FeCN, hexacyanoferrate (III); \\ FeS cluster, iron-sulfur cluster; \\ FMN, flavin mononucleotide; \\ HAR, hexaammineruthenium (III); \\ dNADH, reduced nicotinamide hypoxanthine dinucleotide (deamino-NADH); \\ RCR, respiratory control ratio; \\ ROS, reactive oxygen species.
}




\section{Synopsis}

NADH:ubiquinone oxidoreductase (complex I) is an entry point for electrons into the respiratory chain in many eukaryotes. It couples NADH oxidation and ubiquinone reduction to proton translocation across the mitochondrial inner membrane. Because complex I deficiencies occur in a wide range of neuromuscular diseases, including Parkinson's disease, there is a clear need for model eukaryotic systems to facilitate structural, functional and mutational studies. Here, we describe the purification and characterization of the complexes I from two yeast species, Pichia pastoris and Pichia angusta. They are obligate aerobes which grow to very high cell densities on simple media, as yeast-like, spheroidal cells. Both Pichia enzymes catalyze inhibitor-sensitive NADH:ubiquinone oxidoreduction, display EPR spectra which match closely to those from other eukaryotic complexes I, and show patterns characteristic of complex I in SDS-PAGE analysis. Mass spectrometry was used to identify several canonical complex I subunits. Purified P. pastoris complex I has a particularly high specific activity, and incorporating it into liposomes demonstrates that $\mathrm{NADH}$ oxidation is coupled to the generation of a proton motive force. Interestingly, the rate of $\mathrm{NADH}$-induced superoxide production by the Pichia enzymes is more than twice as fast as that of the Bos taurus enzyme. Our results both resolve previous disagreement about whether Pichia species encode complex I, furthering understanding of the evolution of complex I within dikarya, and they provide two new, robust and highly active model systems for study of the structure and catalytic mechanism of eukaryotic complexes I. 


\section{Introduction}

In mitochondria, NADH:ubiquinone oxidoreductase (complex I) catalyses the oxidation of NADH and the reduction of ubiquinone, coupled to the translocation of four protons across the mitochondrial inner membrane, contributing to the proton motive force [1,2]. Fourteen 'core subunits' are conserved throughout the complex I family. They comprise seven membrane bound subunits, encoded by the mitochondrial genome in eukaryotes, and seven hydrophilic subunits, encoded in the nucleus, which form a domain extending into the mitochondrial matrix. The hydrophilic subunits bind a flavin mononucleotide (FMN), at the active site for NADH oxidation, and eight iron sulfur (FeS) clusters. The structure of the hydrophilic domain from Thermus thermophilus complex I shows how the FeS clusters enable electron transfer from the flavin toward the quinone binding site [3]. In addition, eukaryotic complexes I contain a variable number of 'supernumerary' subunits. Complex I from Bos taurus heart mitochondria has been characterized extensively and contains a total of 45 different subunits [4]. In two fungal model systems, 40 different subunits have been identified in complex I from Yarrowia lipolytica [5], and 39 in the enzyme from Neurospora crassa [6]. Gabaldon and coworkers have used genomic data to propose how the subunit composition of eukaryotic complex I has evolved [7]; mammalian and fungal complexes I have 21 supernumerary subunits in common, while mammals have ten additional subunits, fungi have three. Mutations in both the nuclearly- and mitochondrially-encoded subunits contribute to the increasing number of complex I deficiencies which have been identified in a wide range of neuromuscular diseases, including Leigh syndrome, leukodystrophy, and Parkinson's disease [8,9].

Studies of eukaryotic complex I rely on model systems to provide sufficient quantities of the enzyme for investigation of its structure and mechanism, and to provide insights into the dysfunction of the human enzyme. The B. taurus enzyme is highly homologous to the human enzyme [4], and has proved invaluable in the elucidation of its protein composition, as well as for spectroscopic and functional studies. However, lack of control over the source material, and the impracticality of genetic manipulation, are significant limitations. The intensively studied yeast, Saccharomyces cerevisiae, does not encode complex I, but $N$. crassa [10], and more recently $Y$. lipolytica [11], have provided two genetically manipulable fungal enzymes for study.

Figure 1 shows a phylogenetic tree for the dikarya (adapted from [12]), showing that $N$. crassa belongs to the pezizomycotina, and $Y$. lipolytica to the saccharomycetes. Y. lipolytica diverged relatively early in evolution from the saccharomyceteceae, which include Saccharomyces, Kluveromyces, Candida and Pichia. Some of the saccharomycetaceae have lost the complex I genes during evolution, and, in fact, no complex I has been isolated from any of them. Thus, although the Pichia genus shows many promising characteristics for the development of a complex I model system (see below), it has not been clear, until now, whether Pichia species even express complex I. Sequences for some of the subunits of complex I which are encoded in the mitochondrial genome (known as the ND subunits) have been detected in several species, including $P$. pastoris [13], but attempts to identify an NADH dehydrogenase from $P$. pastoris which is inhibited by a canonical complex I inhibitor, rotenone, were unsuccessful [14]

The two species of yeast described here are Pichia pastoris and Pichia angusta (previously named Hansenula polymorpha). They are both methylotrophic ascomycetes, able to catabolize methanol as their sole carbon source. They are obligate aerobes, and grow on inexpensive, simple media to very high cell densities, particularly in batch-fed conditions. Notably, Pichia species grow almost exclusively as yeast-like, spheroidal cells, whereas $N$. crassa grows as filaments, and $Y$. lipolytica 
switches between yeast and filamentous growth in response to various environmental stresses [15], a transition known to affect cellular metabolism and protein expression [16]. Both species can be used for overexpression, commonly controlled by the promoter for alcohol oxidase, and induced with methanol [17]. In addition, $P$. angusta can grow at temperatures as high as $\sim 50{ }^{\circ} \mathrm{C}$ [18], and therefore may express more stable proteins as part of its heat tolerance. Here, we describe the purification and characterization of the complexes I from $P$. pastoris and $P$. angusta, discuss their suitability as eukaryotic model systems, and compare their properties to those of other members of the complex I family.

\section{Experimental Methods}

All chemicals were from Sigma Aldrich or BDH (unless otherwise stated), and chromatographic media were from GE Healthcare. Buffer $\mathrm{pH}$ values are reported at room temperature.

\section{Yeast strains and growth}

Haploid P. pastoris strain X33 (Invitrogen) was grown at $30{ }^{\circ} \mathrm{C}$ in $1 \%$ yeast extract (ForMedium), 2\% peptone (ForMedium) and 2\% glycerol (VWR International), initially at pH 5. $60 \mathrm{~L}$ of media, in a $70 \mathrm{~L}$ Applikon ADI 1075 fermentor stirred at $500 \mathrm{rpm}$, maintained at $80 \% \mathrm{pO}_{2}$, was inoculated with $2 \mathrm{~L}$ of culture, and cells grown until early stationary phase (approx. 24 hours). P. angusta strain A-16 (NCYC 2310), a haploid leucine auxotroph of CBS4732, was from The National Collection of Yeast Cultures (UK). Cells were grown at $40{ }^{\circ} \mathrm{C}$ in $800 \mathrm{~mL}$ aliquots in $2 \mathrm{~L}$ flasks (shaken at $250 \mathrm{rpm}$ ) until mid stationary phase (approx. 48 hours) in 1\% yeast extract, 2\% peptone, and $4 \%$ glucose, initially at pH 5. Y. lipolytica strain GB10 was provided by Prof. U. Brandt (Frankfurt). It was grown at $27^{\circ} \mathrm{C}$ in $2 \%$ yeast extract, $4 \%$ peptone, and $4 \%$ glucose, initially at pH 5.5, in the $70 \mathrm{~L}$ fermentor stirred at $500 \mathrm{rpm}$, maintained at $2 \% \mathrm{pO}_{2}$ (once this value was reached), until late exponential phase (approx. 20 hours). All cells were harvested by centrifugation (5,000 g for $15 \mathrm{~min}$.), then twice resuspended in MilliQ water and recentrifuged. If necessary, cell pellets were frozen at $-80{ }^{\circ} \mathrm{C}$. The yields were approximately 41, 27 and $29 \mathrm{~g}$ of wet cells per liter of medium for P. pastoris, P. angusta, and $Y$. lipolytica, respectively. All subsequent steps were at $4{ }^{\circ} \mathrm{C}$.

\section{Isolation of mitochondrial membranes}

Methods for cell disruption and membrane isolation were based on those described for $Y$. lipolytica $[19,20]$. Cells were resuspended to $500 \mathrm{~g} \mathrm{~L}^{-1}$, in $20 \mathrm{mM}$ MOPS pH 7.2, $400 \mathrm{mM}$ sorbitol (NBS Biologicals Ltd), 0.2\% BSA, $5 \mathrm{mM}$ EDTA, $1 \mathrm{mM}$ benzamidine, and $1 \mathrm{mM}$-caproic acid. $1 \mathrm{mM}$ phenylmethanesulfonylfluoride (PMSF) was added immediately prior to cell disruption in a DYNOßMILL bead mill (Willy A. Bachofen UK Ltd.) maintained below $10{ }^{\circ} \mathrm{C}$ (two passes at $10 \mathrm{~mL} \mathrm{~min}^{-1}$ ). Cell debris was removed by centrifugation $(5,000 \mathrm{~g}$ for $15 \mathrm{~min}$.), then the membranes were collected by centrifugation (one hour at $100,000 \mathrm{~g}(P$. pastoris and $Y$. lipolytica) or $30,000 \mathrm{~g}$ ( $P$. angusta)), rehomogenized in $20 \mathrm{mM}$ MOPS pH 7.2, $5 \mathrm{mM}$ EDTA, $1 \mathrm{mM}$ benzamidine, and $1 \mathrm{mM} \varepsilon$-caproic acid, and recollected by recentrifugation. If necessary, membrane pellets were frozen at $-80{ }^{\circ} \mathrm{C}$.

\section{Complex I purification}

Mitochondrial membranes were homogenized in $20 \mathrm{mM}$ sodium citrate $\mathrm{pH} 6.5$, to $20 \mathrm{mg}$ protein $\mathrm{mL}^{-1}$, and solubilized for $45 \mathrm{~min}$. by the addition of dodecyl- $\beta$-d-maltoside (DDM, Glycon GmbH) to $2.3 \%$ (P. pastoris) or $2.9 \%$ (P. angusta). Following centrifugation (120,000 g for $45 \mathrm{~min}$.), the supernatant was passed through a MacroCap SP cation exchange column, and dialyzed against $20 \mathrm{mM}$ Tris- $\mathrm{Cl} \mathrm{pH}$ 
7.5 for at least 3 hours. Then, it was loaded onto a Q Sepharose High Performance anion exchange column, pre-equilibrated in $20 \mathrm{mM}$ Tris- $\mathrm{Cl}(\mathrm{pH} 7.5)$ and $0.15 \% \mathrm{DDM}$, and eluted at $2 \mathrm{~mL} \mathrm{~min}^{-1}$. First, $0.27 \mathrm{M} \mathrm{NaCl}$ ( $P$. pastoris) or $0.22 \mathrm{M} \mathrm{NaCl}$ ( $P$. angusta) was applied until the 280 and $420 \mathrm{~nm}$ absorbancies became negligible, then complex I was eluted in a sharp gradient to $0.33 \mathrm{NaCl}(P$. pastoris) or $0.27 \mathrm{M} \mathrm{NaCl}$ ( $P$. angusta), and confirmed using its dNADH:hexaammineruthenium (III) (HAR) oxidoreductase activity. Pooled fractions were concentrated (100 kDa cut-off Vivaspin centrifugal concentrators, Sartorius AG), then injected onto a Superose 6 size exclusion column $(0.24$ $\mathrm{mL} \mathrm{min}^{-1}$, in $20 \mathrm{mM}$ MOPS pH 7.5, 0.05\% DDM and $150 \mathrm{mM} \mathrm{NaCl}$ ). Monomeric complex I eluted from 11 to $14 \mathrm{~mL}$. If necessary, these complex I containing fractions were pooled, concentrated, and reapplied to the column, providing a single, sharp peak. Complex I from Y. lipolytica was isolated as described previously, by affinity purification on a Ni-NTA column using a hexahistidine tag on the Cterminus of the NUGM subunit [20,21], followed by size exclusion chromatography on a Superose 6 column B. taurus complex I was prepared as described previously [22].

\section{Kinetic measurements}

Kinetic measurements were carried out at $32{ }^{\circ} \mathrm{C}$, either in $1 \mathrm{~mL}$ cuvettes (Ocean Optics diode array spectrometer), or in $200 \mu \mathrm{L}$ wells (Molecular Devices microtiter plate reader). Deamino-NADH (dNADH) and NADH were added to $100 \mu \mathrm{M}$ for ferricyanide (hexacyanoferrate (III), FeCN), HAR and decylubiquinone (DQ) measurements, and HAR, FeCN and DQ to $3.5 \mathrm{mM}, 2 \mathrm{mM}$ and $100 \mu \mathrm{M}$, respectively. For measurements using DQ, soybean asolectin was added to $0.35 \mathrm{mg} \mathrm{mL}^{-1}$ from a $10 \mathrm{mg}$ $\mathrm{mL}^{-1}$ stock solution in $20 \mathrm{mM}$ Tris- $\mathrm{HCl}$ pH 7.5 and $1 \%$ w/v CHAPS (3-[(3-cholamidopropyl)dimethylammonio]-1-propane-sulfonate), Anatrace). Rotenone or piericidin A were added to 5 or $2 \mu \mathrm{M}$, respectively, from ethanolic stock solutions. Initial rates of NADH oxidation $\left(\varepsilon_{340-380 \mathrm{~nm}}=4.81 \mathrm{mM}^{-1}\right.$ $\mathrm{cm}^{-1}$ ) were obtained using linear regression. $\mathrm{H}_{2} \mathrm{O}_{2}$ and superoxide production were measured at $\mathrm{pH}$ 7.5, using $30 \mu \mathrm{M}$ NADH and either $10 \mu \mathrm{M}$ Amplex Red and $2 \mathrm{U} \mathrm{mL}^{-1}$ horse radish peroxidase (Invitrogen) or $50 \mu \mathrm{M}$ acetylated cytochrome $c$, as described previously [23].

To produce proteoliposomes containing complex I, $440 \mu \mathrm{L}$ of $10 \mathrm{mg} \mathrm{mL}^{-1}$ soy asolectin (Avanti Polar Lipids Inc.) in $20 \mathrm{mM}$ potassium 3-(N-morpholino) propanesulfonic acid at $\mathrm{pH} 7.5$ (K-MOPS) was homogenised with $60 \mu \mathrm{L}$ of $10 \%$ octyl glucoside. $50 \mu \mathrm{L}$ of $2 \mathrm{mg} \mathrm{mL}^{-1}$ complex I was added, then the detergent was removed by three successive additions of $50 \mathrm{mg}$ Amberlite XAD 2 biobeads (Supelpak) at 1 hour intervals. The proteoliposomes were separated from the biobeads using a syringe fitted with a filter, collected by centrifugation (1 hour at 90,000 $\mathrm{g}$ in a Beckman-Coulter MLA 130 rotor), then resuspended in $100 \mu \mathrm{L}$ K-MOPS. Respiratory control ratios (RCRs) were calculated from the rate of NADH:DQ oxidoreduction, measured (as described above) in the presence and absence of $7 \mu \mathrm{gL}^{-1}$ gramicidin $(\mathrm{RCR}=$ uncoupled rate / coupled rate). The quenching of the 9-amino-6-chloro-2methoxyacridine (ACMA) fluorescence at $475 \mathrm{~nm}$ was measured using an excitation wavelength of $430 \mathrm{~nm}$, in the presence of $500 \mathrm{nM}$ ACMA and $5 \mu \mathrm{M}$ valinomycin. Typically, $20 \mu \mathrm{L}$ of resuspended proteoliposomes were added to $2 \mathrm{~mL}$ of assay buffer. When required carbonylcyanide-ptrifluoromethoxy-phenylhydrazone $(8 \mu \mathrm{M}, \mathrm{FCCP})$ or piericidin A $(2 \mu \mathrm{M})$ were added.

\section{EPR spectroscopy}

EPR samples $\left(\sim 15 \mathrm{mg} \mathrm{mL}^{-1}\right)$ were reduced anaerobically with $5 \mathrm{mM} \mathrm{NADH}$, and frozen immediately. Spectra were recorded on a Bruker EMX X-band spectrometer using an ER 4119HS cavity, maintained at low temperature by an ESR900 continuous-flow liquid helium cryostat (Oxford Instruments); the sample temperature was measured with a calibrated Cernox resistor (Lake Shore Cryotronics Inc.). 


\section{Analytical methods}

Protein concentrations were measured using the Pierce bicinchoninic acid assay. FMN concentrations were analyzed fluorometrically using the method of Burch [24], modified for the plate reader. Iron concentrations were determined using ferene (5,5'(3-(2-pyridyl)-1,2,4-triazine-5,6 diyl)-bis-2furansulfonate), by the method of Pieroni et al. [25] (except the protein was denatured in $1 \% \mathrm{HCl}$ and $1.5 \%$ trichloroacetic acid, the reducing agent was $0.5 \%$ hydroxylamine, and $5 \mathrm{mM}$ thiosemicarbazide was added to bind any copper present).

\section{Protein analyses}

SDS-PAGE was carried out on an 18-22\% acrylamide gradient, and gels were stained with $0.2 \%$ Coomassie Blue R250. Peptide mass fingerprinting and tandem MS were carried out using an Applied Biosystems / MDS SCIEX model 4800 Plus MALDI TOF / TOF mass spectrometer. Database matches to peptide mass fingerprinting and tandem MS data were identified using Mascot (Matrix Science) [26]. Sequences for proposed subunits of $P$. angusta complex I were identified by performing tblastn searches to compare known $Y$. lipolytica and $N$. crassa sequences with the genome survey sequence information from $P$. angusta which is available on the NCBI website.

\section{Results}

\section{The presence of both complex I and alternative NADH dehydrogenases in Pichia species}

Alternative NADH dehydrogenases are single subunit, non-energy transducing NADH:ubiquinone oxidoreductases which are found in many aerobically respiring organisms, either alongside complex I, or, in cases such as $S$. cerevisiae, as a replacement for it (see Figure 1) [27,28]. Alternative NADH dehydrogenases complicate the identification, analysis, and purification of complex I, because the catalytic properties of the two enzymes overlap. Table 1 presents the catalytic properties of mitochondrial membranes prepared from P. pastoris and P. angusta; in both cases approximately $90 \%$ of the NADH binding sites were accessible to the NADH, FeCN and HAR reactants. Three different electron acceptors were used: FeCN and HAR are considered to react at, or close to, the flavin site in complex I, so, unlike DQ, an analogue of the physiological ubiquinone, they do not rely on the functional integrity of the quinone binding site or the membrane domain. First, based on a study of the NADH:ubiquinone oxidoreductases in E. coli, dNADH is assumed to react only with complex I [29], so comparing the rates of $\mathrm{NADH}$ and $\mathrm{dNADH}$ :DQ oxidoreduction by the $P$. pastoris membranes suggests that $\sim 80 \%$ of the NADH:DQ oxidoreductase activity is due to complex I. In contrast, for $P$. angusta the rate with $\mathrm{dNADH}$ is only $\sim 10 \%$ of that with $\mathrm{NADH}$, indicating that one or more alternative NADH dehydrogenases contribute significantly. There is little difference, in either case, between the rates of dNADH and NADH:HAR oxidoreduction, consistent with previous suggestions that HAR does not react with the alternative enzymes [11]. dNADH:FeCN oxidoreduction is significantly faster than dNADH:HAR oxidoreduction in both species, suggesting that FeCN reduction by the Pichia complexes $I$ is much faster than HAR reduction. Furthermore, additional FeCN reductases may be present, as suggested by comparison of the dNADH and NADH:FeCN oxidoreductase activities. Note that the concentrations of $\mathrm{FeCN}$ and HAR used were chosen because they give close to maximal rates for complex I from B. taurus; an extensive investigation of the kinetics of the two Pichia enzymes has not been undertaken so far. Second, NADH:DQ and dNADH:DQ oxidoreduction by the P. pastoris membranes is 85 and $90 \%$ sensitive to the complex I inhibitor piericidin A, consistent with the results described above; the weaker inhibition from rotenone suggests that it is a relatively poor inhibitor in this case. NADH:DQ oxidoreduction by $P$. angusta is not inhibited strongly by either compound, 
although, as expected, the dNADH:DQ activity is more strongly inhibited. Taken together, the results in Table 1 indicate that only 10-20\% of the NADH:DQ oxidoreductase activity from the $P$. angusta membranes is due to complex I. Interestingly, dNADH:HAR oxidoreduction by the $P$. angusta membranes was optimised by harvesting the cells during stationary phase, and by growing them under high aeration. Similar observations were made previously for $P$. jadinii (also known as Torulopsis utilis or Candida utilis) [30,31] and $N$. crassa [32]. In particular, mitochondria from P. jadinii cells harvested in stationary phase displayed piericidin A sensitive NADH oxidation and the complex I EPR signals N1b and N2, but mitochondria from cells harvested in exponential phase did not.

\section{Purification and confirmation of the complexes I from $P$. pastoris and $P$. angusta}

dNADH:HAR oxidoreductase activities were relied upon to guide the development of purification protocols for the two complexes I (see Figure 2). Methods for isolating the complexes I were based on the established protocol for $B$. taurus complex I: solubilization with DDM, anion exchange chromatography, and size exclusion chromatography [22]. Passing the solubilized membranes through a MacroCap cation exchange column, prior to application to the anion exchange column, improved the efficiency of the anion exchange step, particularly for $P$. angusta. For $P$. pastoris, dNADH:HAR oxidoreduction was observed in only one of the major peaks eluting from the anion exchange column $(270$ - $300 \mathrm{mM} \mathrm{NaCl})$. For $P$. angusta, dNADH:HAR oxidoreductase activity eluted in two major peaks, at 50 to $120 \mathrm{mM} \mathrm{NaCl}$, and 220 to $270 \mathrm{mM} \mathrm{NaCl}$. Only the latter peak catalyzed dNADH:DQ oxidoreduction, and the results below confirm it was complex I. Typically, $1 \mathrm{~g}$ of wet cells yielded $12.7 \mu \mathrm{g}$ of $P$. pastoris complex I, or $7.6 \mu \mathrm{g}$ of $P$. angusta complex I. In comparison, $Y$. lipolytica has been reported to yield $\sim 30 \mu \mathrm{g}$ per gram of wet cells by conventional methods [33] or $50-100 \mu \mathrm{g}$ by affinity chromatography [20].

During analytical size exclusion chromatography both Pichia complexes I eluted as single, symmetrical peaks at $11.9 \pm 0.2 \mathrm{~mL}(P$. pastoris) or $12.1 \pm 0.1 \mathrm{~mL}(P$. angusta). The well characterized complexes from $B$. taurus and $Y$. lipolytica eluted at 11.8 and $11.9 \mathrm{~mL}$, respectively. Their protein molecular masses of $\sim 980 \mathrm{kDa}$ [34] and $\sim 940 \mathrm{kDa}$ [5], respectively, were included in a molecular mass calibration to estimate masses for the P. pastoris and $P$. angusta enzymes of 920 and $890 \mathrm{kDa}$, respectively. The FMN and iron contents of the purified enzymes were 1.0 $\pm 0.1 \mathrm{FMN}$ and $33.2 \pm 3.6$ Fe per $P$. pastoris complex I, and $1.1 \pm 0.2 \mathrm{FMN}$ and $33.4 \pm 4.5$ Fe per $P$. angusta complex I, using these estimated molecular masses.

The EPR spectra of both the P. pastoris and P. angusta enzymes (see Figure 3), are typical complex I spectra, and confirm, unambiguously, the identity of the purified enzyme [35]. Comparison with the spectra from B. taurus complex I [35,36] is straightforward, and four signals (N1b, N2, N3 and N4) are immediately identified by their similar g-values. The N5 signal, observed in complex I from B. taurus and Y. lipolytica [33], and the N1a signal, observed in complex I from E. coli [37], are not observed. The slowly relaxing $\mathrm{N} 2$ spectrum, assigned to the [4Fe-4S] cluster in NUKM (PSST) ${ }^{1}$, is evident at around $12 \mathrm{~K}$, with $\mathrm{g}_{\mathrm{z}}$-values matching those from the $B$. taurus and Y. lipolytica enzymes $\left(\mathrm{g}_{\mathrm{z}}=2.06\right.$ for both $P$. pastoris and $P$. angusta). At $40 \mathrm{~K}$ the $\mathrm{N} 1 \mathrm{~b}$ spectrum, from the [2Fe-2S] cluster in NUAM (75 $\mathrm{kDa})$, is observed, again with $\mathrm{g}_{\mathrm{z}}$-values matching those from B. taurus and Y. lipolytica complex I $\left(\mathrm{g}_{\mathrm{z}}=\right.$ 2.03 for both $P$. pastoris and $P$ angusta). At lower temperatures (most clearly 7 and $9 \mathrm{~K}$ ) two further $[4 \mathrm{Fe}-4 \mathrm{~S}]$ spectra are observed. The $\mathrm{g}_{\mathrm{x}}$ value for $\mathrm{N} 3$, assigned to the $[4 \mathrm{Fe}-4 \mathrm{~S}]$ cluster in NUBM (51

\footnotetext{
${ }^{1}$ We name the subunits according to their SwissProt codes, and present the names for the B. taurus homologues alongside where appropriate.
} 
$\mathrm{kDa}$ ), is 1.86 for both $P$. pastoris and $P$. angusta (the $\mathrm{g}_{\mathrm{z}}$ signal cannot be distinguished). The origin of $\mathrm{N} 4$ is controversial $[38,39]$ but the $\mathrm{g}_{\mathrm{z}}$ and $\mathrm{g}_{\mathrm{x}}$ signals of 2.11 and 1.89 (in both cases) match closely to those from $Y$. lipolytica and $B$. taurus complex I.

\section{Catalytic activities of the purified enzymes}

Table 2 summarises the catalytic properties of the complexes I from $P$. pastoris and P. angusta, and compares them with those from $Y$. lipolytica and B. taurus (measured in this study). All the rates of NADH:HAR oxidoreduction are similar, but the rates of NADH:FeCN oxidoreduction are significantly higher for the two Pichia enzymes. Note that our reported values refer to only a single condition, so they are only an indication of the relative catalytic activities of the enzymes, and do not allow the cause of the variation to be identified. Using the structure of the hydrophilic domain of $T$. thermophilus complex I as a template, sequence comparisons for the protein around the NADH binding site in B. taurus, Y. lipolytica, and P. angusta have not provided any insight. The rates of NADH:DQ oxidoreduction are similar in all cases, except for the $P$. pastoris enzyme, which displays a markedly higher rate. Note that the highest published values for $Y$. lipolytica $\left(6-7 \mu \mathrm{mol} \mathrm{min}{ }^{-1} \mathrm{mg}^{-1}\right.$ [40]) exceed those achieved here, but are comparable to our values from $P$. pastoris. Interestingly, all four enzymes are close to completely inhibited by $2 \mu \mathrm{M}$ piericidin $\mathrm{A}$, but the three yeast enzymes are much less effectively inhibited by rotenone than the B. taurus enzyme (see below).

The markedly more active $P$. pastoris complex I could be reconstituted into liposomes, using methods based on those for Y. lipolytica [21] (see Experimental Methods), to provide well coupled proteoliposomes, typically with respiratory control ratios ( $R C R$, the ratio of the rates of NADH oxidation in the presence and absence of an uncoupler) of $\sim 2$. Proteoliposomes formed from the less active $P$. angusta enzyme did not show any coupling $(\mathrm{RCR}=1)$. Figure 4 shows typical traces monitoring the fluorescence of ACMA, a measure of $\triangle \mathrm{pH}$ across the liposomal membrane [41], for reconstituted $P$. pastoris complex I. In the presence of valinomycin (so that potassium ion transport collapses $\Delta \psi$ and facilitates $\Delta \mathrm{pH}$ formation) the ACMA fluorescence is rapidly quenched when catalysis is initiated. It is re-established upon the addition of a proton ionophore, FCCP, to dissipate the proton motive force, or an inhibitor (piericidin A) to prevent further proton translocation. The results shown in Figure 4 are strong evidence for proton translocation by complex I from P. pastoris.

Rates of reactive oxygen species (ROS) generation by the complexes I from both Pichia species were measured as $\mathrm{H}_{2} \mathrm{O}_{2}$ (detected using Amplex Red) or superoxide (detected using acetylated cytochrome $c$ ), and verified by comparison with independent measurements of the rates of NADH oxidation (see Table 2). Within experimental error, the rates of NADH oxidation matched the rates of $\mathrm{H}_{2} \mathrm{O}_{2}$ production in a 1:1 ratio, yalidating both measurements. In both cases, the rates of superoxide formation (one electron) are close to twice the rates of $\mathrm{H}_{2} \mathrm{O}_{2}$ formation (two electrons), indicating that the enzymes from $P$. pastoris and $P$. angusta, like the enzyme from $B$. taurus [23], produce ROS predominantly as superoxide. Table 2 shows that complex I from $Y$. lipolytica falls into the same class, although the rate of superoxide production measured here $\left(90 \mathrm{nmol} \mathrm{min} \mathrm{mg}^{-1}\right.$, validated using the rate of NADH oxidation) is significantly higher than reported previously (18 nmoles $\mathrm{min}^{-1} \mathrm{mg}^{-1}$ [42]). The cause of this discrepancy is not clear. Table 2 shows that the three yeast complexes I produce significantly more ROS than the $B$. taurus enzyme.

\section{The protein composition of the complexes I from $P$. pastoris and $P$. angusta}

Figure 5 shows an SDS PAGE analysis of the protein composition of the complexes I from B. taurus, $Y$. lipolytica, $P$. pastoris and $P$. angusta. The overall similarity of the pattern, particularly for the high molecular weight subunits, is striking, though differences in mass and/or migration become apparent lower down the gel. Bands corresponding to the NUAM (75 kDa), NUBM (51 kDa), NUCM (49 kDa), 
NUDM/NUEM (42/39 kDa) and NUGM (30 kDa) subunits are clearly visible in all cases (NUBM and NUCM comigrate in $Y$. lipolytica), but one of the bands present in the mammalian enzyme, probably the $42 \mathrm{kDa}$ subunit, does not appear in the lanes from the three yeast species.

The complexes I from $B$. taurus and $Y$. lipolytica have already been characterized extensively, using publically available sequence data, but there is little available for $P$. pastoris and $P$. angusta. In order to identify as many subunits as possible in the two Pichia complexes, all the visible protein bands were excised from the gels, digested with trypsin, and subjected to MALDI-TOF and tandem MS analysis. For both $P$. pastoris and $P$. angusta the observed peptide masses (from MALDI-TOF MS) were searched (for matches within $70 \mathrm{ppm}$ ) against the NCBI non-redundant sequence database, and against an in-house database of expected complex I subunits from P. angusta (derived from the NCBI genome survey sequences by comparison with known sequences from other species). Then, the closest matches to the tandem MS data (within $120 \mathrm{ppm}$ ) were re-evaluated using error tolerance searches, allowing for modifications and amino acid substitutions between species. A summary of the data assigned is presented in Supplementary Table S1. All seven hydrophilic core subunits, and two supernumerary subunits, were identified in the enzyme from P. pastoris. Six of the hydrophilic core subunits (the exception being NUKM (PSST)), and nine supernumerary subunits were identified in the enzyme from $P$. angusta. The subunits of $P$. pastoris complex I were identified using the similarity of observed peptide masses to predicted peptide masses from other fungal species, including $P$. stipitis (NUAM (75 kDa), NUBM (51 kDa), NUCM (49 kDa), NUEM (39 kDa), NUGM (30 kDa), NUHM (24 kDa), NUYM (18 kDa), NUIM (TYKY) and NUKM (PSST)), Lodderomyces elongisporus (NUAM), Debaryomyces hansenii (NUBM, NUCM, and NUHM), Y. lipolytica (NUBM, NUCM, and NUIM), $P$. guilleromondii (NUEM), N. crassa (NUKM), Aspergillus fumigatus (NUKM), and Candida albicans (NUYM). These matches confirm that $P$. pastoris complex I contains a number of canonical complex I subunits, but the lack of complete sequences for them precludes further discussion about its subunit composition and homology to other species. The genome sequence for $P$. pastoris is available commercially, and the composition of its complex I will be described in detail in a future publication. In the same way, masses from five subunits in $P$. angusta complex I were found to be similar to those in other species: P. stipitis (NUAM (75 kDa), and NUCM (49 kDa)), N. crassa (NUBM (51 kDa)), D. hansenii (NUCM) Dekkera bruxellensis (NUHM (24 kDa)), Magnaporthe grisea (NUHM), and $A$. niger (NB6M (B16.6)). For $P$. angusta there are several full or partial sequences available publically as genome survey sequences, and they allowed the identification of eleven complex I subunit sequences (NUBM (51 kDa), NUGM (30 kDa), NUIM (TYKY), NUZM, NIAM (ASHI), N7BM (B17.2), NUKM (B14.7), NUFM (B13), NUMM (13 kDa), NIMM (MWFE) and NIDM (PDSW)). A comparison of observed peptide masses from $P$. pastoris against these $P$. angusta sequences only provided a match to one complex I subunit (NUIM (TYKY)).

\section{Discussion}

\section{The presence of complex I in Pichia species}

The data presented here show that both $P$. pastoris and $P$. angusta assemble a piericidin A sensitive, multisubunit NADH:ubiquinone oxidoreductase in their mitochondria, with closely similar catalytic and spectroscopic properties to the enzymes from B. taurus and $Y$. lipolytica. A number of the core subunits of complex I have been identified in both Pichia species, along with several supernumerary subunits, which vary between kingdoms, subkingdoms and between genera and species also [7]. Our data and preliminary sequence comparisons suggest that, within the species included in Figure 1, 
complex I from $P$. pastoris is most closely related to the enzyme from $P$. stipitis, whereas $P$. angusta complex I is most closely related to the enzymes from $Y$. lipolytica, L. elongisporus, $P$. stipitis, $P$. guilleromondii and $D$. hansenii (but not to any one of them in particular).

The presence of the complex I ND genes in the mitochondrial genomes of many fungi (including Pichia species) [13,28], suggests that most (if not all) obligate aerobic fungi are able to express complex I. Those species capable of facultative anaerobic fermentation encode only the single subunit enzyme (see Figure 1). Without a larger set of sequences for comparison, it is difficult to speculate further on the evolutionary development of complex I in some species, and its loss in others. However, intriguing correlations can be identified within the species in Figure 1. Only species that encode complex I encode the alternative, single subunit, non-proton pumping ubiquinol oxidase [28]: if complexes III and IV are substituted by the alternative oxidase, complex I is retained in order to maintain the proton motive force (or vice versa). Only species in the subphylum pezizomycota encode the complex I supernumerary subunit NURM [28], and they are the only species that encode the three subunit, proton pumping transhydrogenase also. In $N$. crassa NURM is a $17.8 \mathrm{kDa}$ protein containing one transmembrane helix, with no known homology to any other protein.

\section{The catalytic activities of the complexes I from Pichia species}

The preparation of a highly pure eukaryotic complex I with native NADH:ubiquinone oxidoreductase activity has proved challenging, particularly for the mammalian complexes. Recently, by including phospholipids in the preparation, highly pure complex I has been prepared from $B$. taurus mitochondria with NADH:DQ oxidoreductase activity up to $4 \mu \mathrm{mol} \mathrm{NADH} \mathrm{min}^{-1} \mathrm{mg}^{-1}$ [22]. For Y. lipolytica, preparations with an activity of $\sim 2.8 \mu \mathrm{mol} \mathrm{min} \mathrm{mg}^{-1}$ were obtained by conventional purification [33], but a histidine tag on the NUGM (30 kDa) subunit increased the activity to $6-7 \mu \mathrm{mol} \mathrm{min} \mathrm{mg}^{-1}$ [40]. Here, conventional methods were used to purify the two Pichia complexes I. Initially, it was hoped that the $P$. angusta enzyme would be more stable because of the organism's thermotolerance [18], but the $P$. pastoris enzyme gave a significantly higher activity than that of $P$. angusta $(6.3$ and $4.0 \mu \mathrm{mol}$ NADH min $^{-1} \mathrm{mg}^{-1}$, respectively). It is possible that phospholipid content is key to understanding the relative activities. The $B$. taurus enzyme is readily depleted of phospholipids during preparation [22]. The $P$. angusta enzyme requires more exogenous phospholipids in activity assays than the $P$. pastoris enzyme, to attain its highest activity, and it cannot be incorporated properly into liposomes. It is possible that this stems from its lower relative abundance in the membranes - it must be washed more extensively on the anion exchange column, a procedure which is known to 'strip' the B. taurus enzyme [22]. Finally, variations in the DQ reductase activity are not reflected in any other activity measurements (see Table 2); in particular, NADH:HAR oxidoreduction is essentially the same in all four cases. Previously, a study of the complexes I from B. taurus and P. denitrificans also found that, while FeCN was reduced relatively slowly by the prokaryotic enzyme, the HAR reduction rates were similar [43].

'Tests' which distinguish complex I from other NADH dehydrogenases, and inhibitor sensitivity It is widely assumed that dNADH reacts only with complex I, not with alternative NADH dehydrogenases, although this fact has only been demonstrated in E. coli [29]. All the complexes I tested here (Table 2) react at comparable rates with dNADH and NADH. However, if the dNADH:HAR and dNADH:DQ oxidoreductase activities in the membranes are only from complex I, then the ratio between them should be conserved throughout the preparation. In fact, the ratio (HAR / DQ) increases from 1.1 ( $P$. pastoris) and 5.0 ( $P$. angusta) in the membranes to 9.8 and 21.1, respectively. Because the specific dNADH:HAR oxidoreductase activity is unlikely to vary significantly, the results indicate at least one additional enzyme in the membrane preparations catalyses 
dNADH:DQ oxidoreduction also (or a significant portion of the dNADH:DQ activity has been lost). Consequently, dNADH may differentiate less clearly between complex I and alternative NADH dehydrogenases than commonly supposed. It is widely assumed that only complex I catalyses NADH:HAR oxidoreduction also (see, for example [11]) but we have been unable to find any published data to support this fact. During the preparation of $P$. angusta complex I, two fractions with NADH:HAR oxidoreductase activity eluted from the anion exchange column, but only the second catalysed dNADH:DQ oxidoreduction. Although the protein composition of the first peak was complex, SDS PAGE, mass spectrometry and Western blotting analyses detected no evidence for complex I or any portion it, suggesting that this second 'test' for complex I is also not definitive.

Table 2 shows that all the NADH:DQ oxidoreductase activities tested are strongly inhibited by piericidin A, but rotenone does not inhibit the three yeast enzymes as strongly as the B. taurus enzyme. This observation may explain why rotenone-sensitive NADH oxidation was not observed previously in $P$. pastoris mitochondria [14]. Studies of the inhibition of the activity of $P$. pastoris complex I over a range of rotenone concentrations showed that almost complete inhibition can be observed, but only at much higher concentrations ( $96 \%$ in $50 \mu \mathrm{M}$ rotenone). Relatively weak inhibition of $Y$. lipolytica complex I was observed previously also [42]. Thus, rotenone is regarded as an archetypal complex I inhibitor simply because of the predominance of the $B$. taurus enzyme in early studies.

\section{The production of reactive oxygen species}

Previously, we described a mechanism for ROS production by the reduced flavin in complex I from $B$. taurus, and demonstrated the same mechanism in complex I from E. coli $[23,44]$. Here, we compare ROS production by the complexes I from $P$. pastoris, $P$. angusta and $Y$. lipolytica with that by the $B$. taurus enzyme. Values for all three yeast species were significantly higher than those from $B$. taurus, and those for the two Pichia species were the highest. This is particularly interesting because B. taurus and E. coli complex I exhibit similar rates [44]. When the two Pichia species are grown on methanol the induction of alcohol oxidases results in significant $\mathrm{H}_{2} \mathrm{O}_{2}$ production, which is metabolised by catalase and cytochrome $c$ peroxidase $[17,45]$. The additional contribution from complex I may not be significant in this context. Furthermore, all four eukaryotic enzymes produce predominantly superoxide, not $\mathrm{H}_{2} \mathrm{O}_{2}$. This observation maintains the correlation between superoxide to $\mathrm{H}_{2} \mathrm{O}_{2}$ ratio and the reduction of cluster N1a described previously [44]: the N1a EPR signal from the [2Fe-2S] cluster in NUHM $(24 \mathrm{kDa})$ is not observed in any of the eukaryotic enzymes described here (see Figure 3 ). Therefore, the cluster is predominantly oxidized in the presence of $\mathrm{NADH}$, and is, in principle, available to transiently accept the second electron from the fully reduced flavin, promoting the escape of nascent superoxide from the active site.

\section{Acknowledgements}

We thank Professor Ulrich Brandt (Frankfurt) for providing the strain of Y. lipolytica, Dr. Ian M. Fearnley (MRC) for advice on mass spectrometry, and Martin King (MRC) for providing B. taurus complex I. This work was supported by The Medical Research Council. 


\section{References}

1. Brandt, U. (2006) Energy converting NADH:quinone oxidoreductase (complex I). Annu. Rev. Biochem. 75, 69-92

2. Hirst, J. (2005) Energy transduction by respiratory complex I - an evaluation of current knowledge. Biochem. Soc. Trans. 33, 525-529

3. Sazanov, L. A., and Hinchliffe, P. (2006) Structure of the hydrophilic domain of respiratory complex I from Thermus thermophilus. Science 311, 1430-1436

4. Hirst, J., Carroll, J., Fearnley, I. M., Shannon, R. J., and Walker, J. E. (2003) The nuclear encoded subunits of complex I from bovine heart mitochondria. Biochim. Biophys. Acta 1604, $135-150$

5. Morgner, N., Zickermann, V., Kerscher, S., Wittig, I., Abdrakhmanova, A., Barth, H.-D., Brutschy, B., and Brandt, U. (2008) Subunit mass fingerprinting of mitochondrial complex I. Biochim. Biophys. Acta 1777, 1384-1391

6. Marques, I., Duarte, M., Assunção, J., Ushakova, A. V., and Videira, A. (2005) Composition of complex I from Neurospora crassa and disruption of two accessory subunits. Biochim. Biophys. Acta 1707, 211-220

7. Gabaldón, T., Rainey, D., and Huynen, M. A. (2005) Tracing the evolution of a large protein complex in the eukaryotes, NADH:ubiquinone oxidoreductase (complex I). J. Mol. Biol. 348, $857-870$

8. DiMauro, S., and Schon, E. A. (2003) Mitochondrial respiratory-chain diseases. New Engl. J. Med. 348, 2656-2668

9. Greenamyre, J. T., and Hastings, T. G. (2004) Parkinson's - divergent causes, convergent mechanisms. Science 304, 1120-1122

10. Videira, A. (1998) Complex I from the fungus Neurospora crassa. Biochim. Biophys. Acta 1364, 89-100

11. Kerscher, S., Dröse, S., Zwicker, K., Zickermann, V., and Brandt, U. (2002) Yarrowia lipolytica, a yeast genetic system to study mitochondrial complex I. Biochim. Biophys. Acta 1555, 83-91

12. Kriventseva, E. V., Rahman, N., Espinosa, O., and Zdobnov, E. M. (2008) OrthoDB: the hierarchical catalog of eukaryotic orthologs. Nucleic Acids Res. 36, D271-D275

13. Nosek, J., and Fukuhara, H. (1994) NADH dehydrogenase subunit genes in the mitochondrial DNA of yeasts. J. Bacteriol. 176, 5622-5630

14. González-Barroso, M. M., Ledesma, A., Lepper, S., Pérez-Magán, E., Zaragoza, P., and Rial, E. (2006) Isolation and bioenergetic characterization of mitochondria from Pichia pastoris. Yeast 23, 307-313

15. Ruiz-Herrera, J., and Sentandreu, R. (2002) Different effectors of dimorphism in Yarrowia lipolytica. Arch. Microbiol. 178, 477-483

16. Morín, M., Monteoliva, L., Insenser, M., Gil, C., and Domínguez, A. (2007) Proteomic analysis reveals metabolic changes during yeast to hypha transition in Yarrowia lipolytica. J. Mass Spectrom. 42, 1453-1462

17. Gellissen, G., Kunze, G., Gaillardin, C., Cregg, J. M., Berardi, E., Veenhuis, M., and van der Klei, I. (2005) New yeast expression platforms based on methylotrophic Hansenula polymorpha and Pichia pastoris and on dimorphic Arxula adeninivorans and Yarrowia lipolytica - a comparison. FEMS Yeast Res. 5, 1079-1096

18. van Uden, N., Abranches, P., and Cabeça-Silva, C. (1968) Temperature functions of thermal death in yeasts and their relation to the maximum temperature for growth. Arch. Mikrobiol. 61, 381-393 
19. Kerscher, S. J., Okun, J. G., and Brandt, U. (1999) A single external enzynme confers alternative NADH:ubiqinone oxidoreductase activity in Yarrowia lipolytica. J. Cell Sci. 112, 2347-2354

20. Kashani-Poor, N., Kerscher, S., Zickermann, V., and Brandt, U. (2001) Efficient large scale purification of his-tagged proton translocating NADH:ubiquinone oxidoreductase (complex I) from the strictly aerobic yeast Yarrowia lipolytica. Biochim. Biophys. Acta 1504, 363-370

21. Dröse, S., Galkin, A., and Brandt, U. (2005) Proton pumping by complex I from Yarrowia lipolytica reconstituted into proteoliposomes. Biochim. Biophys. Acta 1710, 87-95

22. Sharpley, M. S., Shannon, R. J., Draghi, F., and Hirst, J. (2006) Interactions between phospholipids and NADH:ubiquinone oxidoreductase (complex I) from bovine mitochondria. Biochemistry 45, 241-248

23. Kussmaul, L., and Hirst, J. (2006) The mechanism of superoxide production by NADH:ubiquinone oxidoreductase (complex I) from bovine heart mitochondria. Proc. Natl. Acad. Sci. USA 103, 7607-7612

24. Burch, H. B. (1957) Fluorimetric assay of FAD, FMN and riboflavin. Methods Enzymol. 3, 960-962

25. Pieroni, L., Khalil, L., Charlotte, F., Poynard, T., Piton, A., Hainque, B., and Imbert-Bismut, F. (2001) Comparison of bathophenanthroline sulfonate and ferene as chromogens in colorimetric measurement of low hepatic iron concentration. Clin. Chem. 47, 2059-2061

26. Perkins, D. N., Pappin, D. J. C., Creasy, D. M., and Cottrell, J. S. (1999) Probability-based protein identification by searching sequence databases using mass spectrometry data. Electrophoresis 20, 3551 - 3567

27. Melo, A. M. P., Bandeiras, T. M., and Teixeira, M. (2004) New insights into type II NAD(P)H: quinone oxidoreductases. Microbiol. Mol. Biol. R. 68, 603-616

28. Lavín, J. L., Oguiza, J. A., Ramírez, L., and Pisabarro, A. G. (2008) Comparative genomics of the oxidative phosphorylation system in fungi. Fungal Genet. Biol. 45, 1248-1256

29. Matsushita, K., Ohnishi, T., and Kaback, H. R. (1987) NADH-ubiquinone oxidoreductases of the Escherichia coli aerobic respiratory chain. Biochemistry 26, 7732-7737

30. Katz, R., Kilpatrick, L., and Chance, B. (1971) Aquisition and loss of rotenone sensitivity in Torulopsis utilis. Eur. J. Biochem. 21,301-307

31. Grossman, S., Cobley, J. G., Singer, T. P., and Beinert, H. (1974) Reduced nicotinamide adenine dinucleotide dehydrogenase, piericidin sensitivity, and site 1 phosphorylation in different growth phases of Candida utilis. J. Biol. Chem. 249, 3819-3826

32. Schwitzguébel, J.-P., and Palmer, J. M. (1982) Properties of mitochondria as a function of the growth stages of Neurospora crassa. J. Bacteriol. 149, 612-619

33. Djafarzadeh, R., Kerscher, S., Zwicker, K., Radermacher, M., Lindahl, M., Schägger, H., and Brandt, U. (2000) Biophysical and structural characterization of proton-translocating NADHdehydrogenase (complex I) from the strictly aerobic yeast Yarrowia lipolytica. Biochim. Biophys. Acta 1459, 230-238

34. Carroll, J. Fearnley, I. M., Skehel, J. M., Shannon, R. J., Hirst, J., and Walker, J. E. (2006) Bovine complex I is a complex of forty-five different subunits. J. Biol. Chem. 281, 3272432727

35. Ohnishi, T. (1998) Iron-sulphur clusters/semiquinones in complex I. Biochim. Biophys. Acta 1364, 186-206

36. Reda, T., Barker, C. D., and Hirst, J. (2008) Reduction of the iron-sulfur clusters in mitochondrial NADH:ubiquinone oxidoreductase (complex I) by Eu ${ }^{\text {II }}$-DTPA, a very low potential reductant. Biochemistry 47, 8885-8893 
37. Uhlmann, M., and Friedrich, T. (2005) EPR signals assigned to Fe/S cluster N1c of the Escherichia coli NADH:ubiquinone oxidoreductase derive from cluster N1a. Biochemistry 44, 1653-1658

38. Yakovlev, G., Reda, T., and Hirst, J. (2007) Reevaluating the relationship between EPR spectra and enzyme structure for the iron-sulfur clusters in NADH:quinone oxidoreductase. Proc. Natl. Acad. Sci. USA 104, 12720-12725

39. Ohnishi, T., and Nakamaru-Ogiso, E. (2008) Were there any misassignments among iron-sulfur clusters N4, N5 and N6b in NADH-quinone oxidoreductase (complex I). Biochim. Biophys. Acta 1777, 703-710

40. Dröse, S., Zwicker, K., and Brandt, U. (2002) Full recovery of the NADH:ubiquinone activity of complex I (NADH:ubiquinone oxidoreductase) from Yarrowia lipolytica by the addition of phospholipids. Biochim. Biophys. Acta 1556, 65-72

41. Dufour, J.-P., Goffeau, A., and Tsong, T. Y. (1982) Active proton uptake in lipid vesicles reconstituted with the purified yeast plasma membrane ATPase. J. Biol. Chem. 257, 9365-9371

42. Galkin, A., and Brandt, U. (2005) Superoxide radical formation by pure complex I (NADH:ubiquinone oxidoreductase) from Yarrowia lipolytica. J. Biol. Chem. 280, 3012930135

43. Zickermann, V., Kurki, S., Kervinen, M., Hassinen, I., and Finel, M. (2000) The NADH oxidation domain of complex I: do bacterial and mitochondrial enzymes catalyze ferricyanide reduction similarly? Biochim. Biophys. Acta 1459, 61-68

44. Esterházy, D., King, M. S., Yakovlev, G., and Hirst, J. (2008) Production of reactive oxygen species by complex I (NADH:ubiquinone oxidoreductase) from Escherichia coli and comparison to the enzyme from mitochondria. Biochemistry 47, 3964-3971

45. Duff, S. J. B., and Murray, W. D. (1990) Metabolism of hydrogen peroxide by Pichia pastoris. Agric. Biol. Chem. 54, 1967-1973 


\section{Tables}

Table 1. Specific activities of the mitochondrial membranes from P. pastoris and P. angusta.

\begin{tabular}{|c|c|c|}
\hline & \multicolumn{2}{|c|}{$\begin{array}{c}\text { Catalytic activity } \\
\left(\mu \mathrm{mol} \text { NADH } \min ^{-1} \mathrm{mg}^{-1}\right)\end{array}$} \\
\hline & P. pastoris & P. angusta \\
\hline NADH:FeCN oxidoreduction & $9.39 \pm 0.56$ & $9.65 \pm 1.02$ \\
\hline dNADH:FeCN oxidoreduction & $6.85 \pm 0.18$ & $6.88 \pm 0.88$ \\
\hline NADH:HAR oxidoreduction & $1.00 \pm 0.07$ & $1.41 \pm 0.18$ \\
\hline dNADH:HAR oxidoreduction & $1.14 \pm 0.07$ & $1.34 \pm 0.14$ \\
\hline NADH:DQ oxidoreduction & $1.27 \pm 0.07$ & $2.16 \pm 0.40$ \\
\hline dNADH:DQ oxidoreduction & $1.00 \pm 0.07$ & $0.27 \pm 0.14$ \\
\hline $\begin{array}{c}\text { NADH:DQ oxidoreduction + } \\
\text { piericidin A }\end{array}$ & $\begin{array}{c}0.19 \pm 0.06 \\
(85 \%)\end{array}$ & $\begin{array}{l}1.63 \pm 0.31 \\
(25 \%)\end{array}$ \\
\hline $\begin{array}{c}\text { dNADH:DQ oxidoreduction + } \\
\text { piericidin A }\end{array}$ & $\begin{array}{c}0.10 \pm 0.04 \\
(90 \%)\end{array}$ & $\begin{array}{l}0.10 \pm 0.01 \\
\quad(63 \%)\end{array}$ \\
\hline $\begin{array}{c}\text { NADH:DQ oxidoreduction + } \\
\text { rotenone }\end{array}$ & $\begin{array}{c}0.95 \pm 0.52 \\
(26 \%)\end{array}$ & $\begin{array}{l}1.71 \pm 0.40 \\
(21 \%)\end{array}$ \\
\hline $\begin{array}{c}\mathrm{dNADH}: \mathrm{DQ} \text { oxidoreduction + } \\
\text { rotenone }\end{array}$ & $\begin{array}{l}0.28 \pm 0.03 \\
(72 \%)\end{array}$ & $\begin{array}{l}0.12 \pm 0.02 \\
(56 \%)\end{array}$ \\
\hline Accessibility of NADH binding sites & $86.2 \pm 1.8 \%$ & $87.1 \pm 8.3 \%$ \\
\hline
\end{tabular}

Conditions: $20 \mathrm{mM}$ Tris-HCl pH 7.5, $100 \mu \mathrm{M}$ NADH or dNADH, $100 \mu \mathrm{M}$ DQ, $3.5 \mathrm{mM}$ HAR, $2 \mathrm{mM}$ $\mathrm{FeCN}, 5 \mu \mathrm{M}$ rotenone, $2 \mu \mathrm{M}$ piericidin $\mathrm{A}, 32{ }^{\circ} \mathrm{C}$. The accessibility of the NADH binding sites in the two preparations was determined from the average increase in the rates of dNADH:HAR, dNADH:FeCN and NADH:FeCN oxidoreduction, following solubilisation of the membranes in $3 \%$ DDM. 
Table 2. Specific activities of the complexes I purified from $P$. pastoris and $P$. angusta, and comparison with the enzymes from $Y$. lipolytica and $B$. taurus.

\begin{tabular}{|c|c|c|c|c|}
\hline \multirow{2}{*}{} & \multicolumn{4}{|c|}{ Catalytic activity $\left(\mu \mathrm{mol}\right.$ NADH $\left.\mathrm{min}^{-1} \mathrm{mg}^{-1}\right)$} \\
\cline { 2 - 5 } & P. pastoris & P. angusta & Y. lipolytica & B. taurus \\
\hline NADH:FeCN oxidoreduction & $302.6 \pm 22.9$ & $248.9 \pm 22.9$ & $140.8 \pm 3.1$ & $111.1 \pm 8.3$ \\
\hline NADH:HAR oxidoreduction & $70.5 \pm 9.4$ & $63.4 \pm 3.2$ & $68.8 \pm 13$ & $72.5 \pm 6.8$ \\
\hline dNADH:HAR oxidoreduction & $68.6 \pm 7.4$ & $69.0 \pm 5.3$ & - & - \\
\hline NADH:DQ oxidoreduction & $6.27 \pm 0.38$ & $4.00 \pm 0.04$ & $3.80 \pm 0.55$ & $3.82 \pm 0.17$ \\
\hline dNADH:DQ oxidoreduction & $7.00 \pm 0.86$ & $3.27 \pm 0.26$ & $3.55 \pm 0.40$ & $3.62 \pm 0.50$ \\
\hline dNADH:DQ oxidoreduction + & $0.37 \pm 0.30$ & $0.06 \pm 0.01$ & $0.28 \pm 0.02$ & $0.24 \pm 0.11$ \\
piericidin A & $(95 \%)$ & $(98 \%)$ & $(92 \%)$ & $(93 \%)$ \\
\hline dNADH:DQ oxidoreduction + & $2.02 \pm 0.53$ & $0.56 \pm 0.11$ & $1.31 \pm 0.67$ & $0.14 \pm 0.01$ \\
rotenone & $(71 \%)$ & $(83 \%)$ & $(63 \%)$ & $(96 \%)$ \\
\hline & \multicolumn{3}{|c|}{$\mathrm{H}_{2} \mathrm{O}_{2}$ or superoxide production $\left.(\mathrm{nmol} \mathrm{min})^{-1} \mathrm{mg}^{-1}\right)$} \\
\hline $\mathrm{H}_{2} \mathrm{O}_{2}$ & $63.3 \pm 4.9$ & $76.3 \pm 14.7$ & $42.5 \pm 4.4$ & $23.6 \pm 5.6$ \\
\hline superoxide & $105.0 \pm 11.6$ & $127.2 \pm 7.5$ & $90.3 \pm 2.8$ & $41.3 \pm 1.8$ \\
\hline NADH:O $\mathrm{O}_{2}$ oxidoreduction & $55.1 \pm 5.8$ & $68.6 \pm 15.6$ & $41.3 \pm 1.8$ & $20.1 \pm 1.7$ \\
\hline
\end{tabular}

Conditions: $20 \mathrm{mM}$ Tris- $\mathrm{HCl} \mathrm{pH}$ 7.5, $100 \mu \mathrm{M}$ NADH, $100 \mu \mathrm{M}$ DQ, $3.5 \mathrm{mM}$ HAR, $2 \mathrm{mM}$ FeCN, $5 \mu \mathrm{M}$ rotenone, $2 \mu \mathrm{M}$ piericidin $\mathrm{A}, 32^{\circ} \mathrm{C}$. $\mathrm{H}_{2} \mathrm{O}_{2}$ measurements: $20 \mathrm{mM}$ Tris- $\mathrm{HCl} \mathrm{pH} 7.5,30 \mu \mathrm{M}$ NADH, 10 $\mu \mathrm{M}$ Amplex Red, $2 \mathrm{U} \mathrm{ml}^{-1}$ horse radish peroxidase. Superoxide measurements: $20 \mathrm{mM}$ Tris- $\mathrm{HCl} \mathrm{pH}$ 7.5, $30 \mu \mathrm{M} \mathrm{NADH}, 50 \mu \mathrm{M}$ partially acetylated cytochrome $c$. Values for $Y$. lipolytica and B. taurus were measured alongside those for $P$. pastoris and $P$. angusta for direct comparison. 


\section{Figure legends}

Figure 1. Phylogenetic tree showing the distribution of complex I within the subkingdom of dikarya. Species in bold have complex I subunits encoded in their nuclear and mitochondrial genomes, and they encode an alternative oxidase and at least one alternative, single subunit NADH:ubiquinone oxidoreductase. Species in grey are able to anaerobically ferment, and they only encode alternative NADH:ubiquinone oxidoreductases. $\left(^{*}\right)$ denotes the presence of the genes for the three subunit mitochondrial transhydrogenase, and the NURM gene, which has been observed previously only in N. crassa complex I. The phylogenetic tree was adapted from [12].

Figure 2. Variation of the specific dNADH:HAR oxidoreductase activity during preparation of the complexes I from $\boldsymbol{P}$. pastoris and $\boldsymbol{P}$. angusta. Memb., mitochondrial membranes; Sol. Memb., membranes following solubilization in DDM; QS, pooled fractions from the Q-sepharose ion exchange chromatography step; SE2, pooled fractions from the second size exclusion chromatography step.

Figure 3. EPR spectra showing signals from the FeS clusters in the complexes I from $P$. pastoris and $\boldsymbol{P}$. angusta reduced by NADH. Spectra are presented at different temperatures because each cluster signal is only observed over a limited range. The signals have been identified, and labeled, by matching their g-values and temperature dependence to those from the well characterized complexes I from $B$. taurus and $Y$. lipolytica. Conditions: $\sim 15 \mathrm{mg} \mathrm{mL}^{-1}$ complex I, $5 \mathrm{mM}$ NADH, $20 \mathrm{mM}$ MOPS $\mathrm{pH} 7.5,150 \mathrm{mM} \mathrm{NaCl}$ and $0.02 \%$ DDM; microwave power $1 \mathrm{~mW}$, conversion time $81.92 \mathrm{~ms}$, time constant $20.48 \mathrm{~ms}$, modulation amplitude $10 \mathrm{G}$, microwave frequency $\sim 9.38 \mathrm{MHz}$.

Figure 4. Proton pumping by complex I from $\boldsymbol{P}$. pastoris reconstituted into liposomes. The formation of $\Delta \mathrm{pH}$ was monitored by the fluorescence of ACMA, which is quenched upon accumulation into the lumen of the liposomes. A small decrease in fluorescence, not related to the formation of $\Delta \mathrm{pH}$, is observed upon the addition of DQ. Significant quenching ( $\Delta \mathrm{pH}$ formation) is observed in the presence of both NADH and DQ, and is dissipated by the presence of either the proton ionophore FCCP, or the complex I inhibitor piericidin A. The potassium ionophore, valinomycin, is present to collapse $\Delta \psi$ and allow $\Delta \mathrm{pH}$ formation. The traces all begin at zero fluorescence intensity and have been offset from one another along the y-axis. Conditions: $100 \mu \mathrm{M}$ NADH, $100 \mu \mathrm{M} \mathrm{DQ}, 500 \mathrm{nM}$ ACMA, $5 \mu \mathrm{M}$ valinomycin, $20 \mathrm{mM}$ MOPS pH 7.5 and $50 \mathrm{mM} \mathrm{KCl}$, excitation $430 \mathrm{~nm}$, emission 475 $\mathrm{nm}$.

Figure 5. Analysis of the subunit composition of the complexes I from $P$. pastoris and $P$. angusta, and comparison with the enzymes from $\boldsymbol{B}$. taurus and $\boldsymbol{Y}$. lipolytica. SDS-PAGE analysis was performed on a $12-22 \%$ gradient gel, and the bands excised and analyzed by peptide mass fingerprinting and tandem mass spectrometry. Peptides were identified using publicly available genome sequences (see Supplementary Table S1). 


\section{Figure 1}

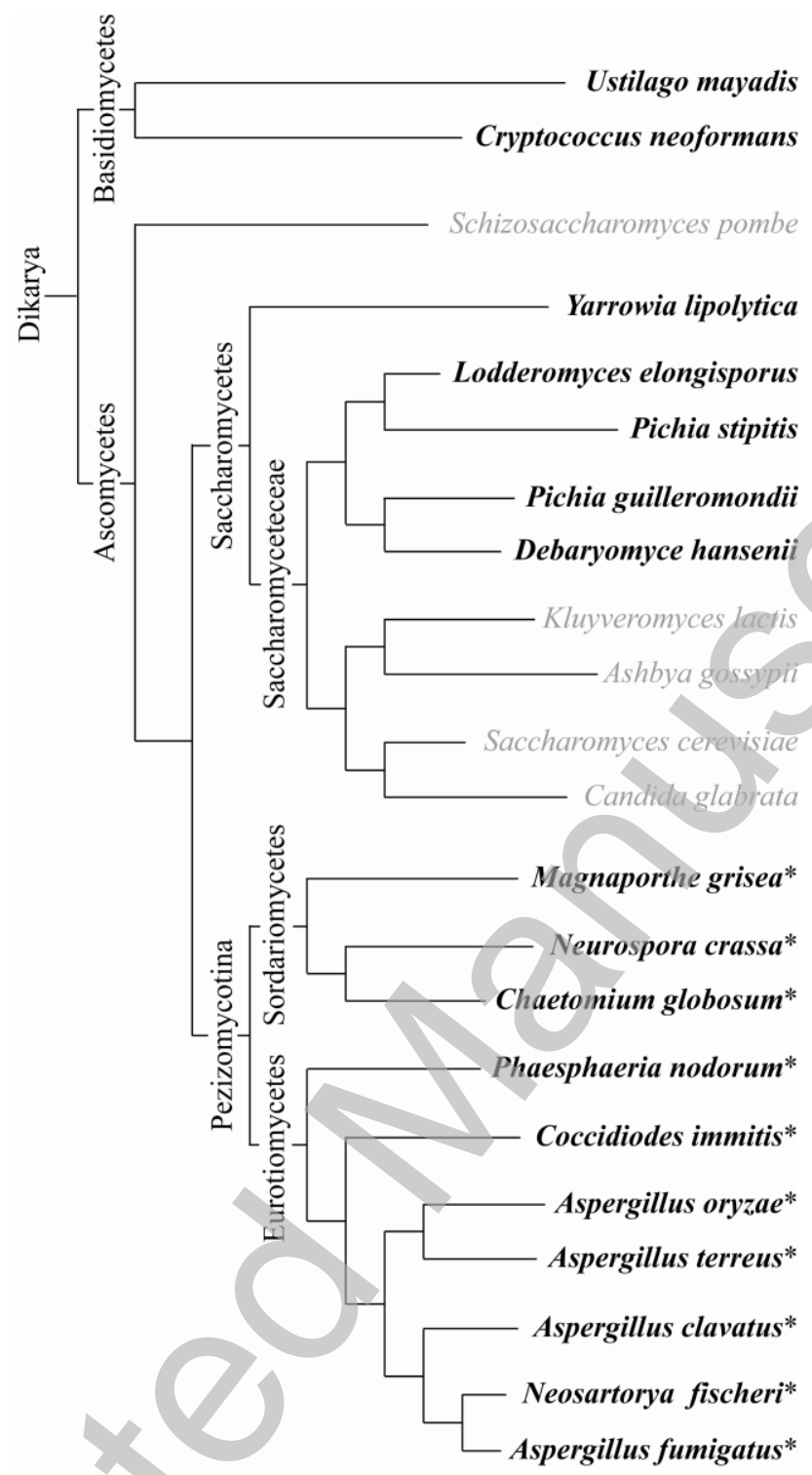




\section{Figure 2}

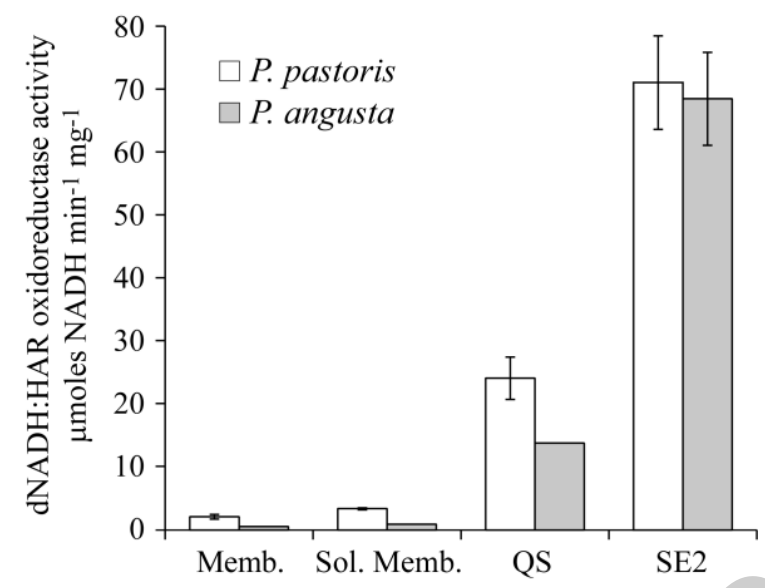

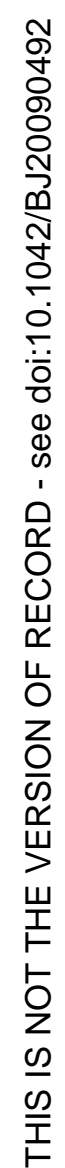




\section{Figure 3}

\section{A. P. pastoris}

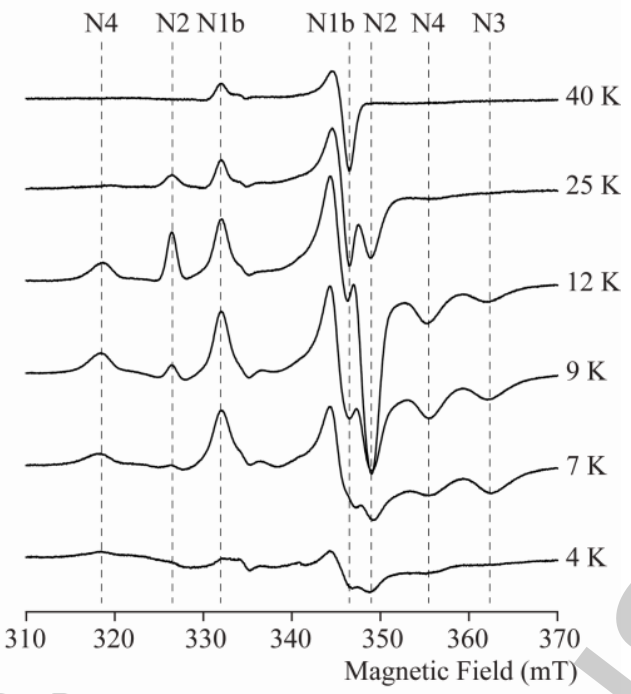

B. P. angusta

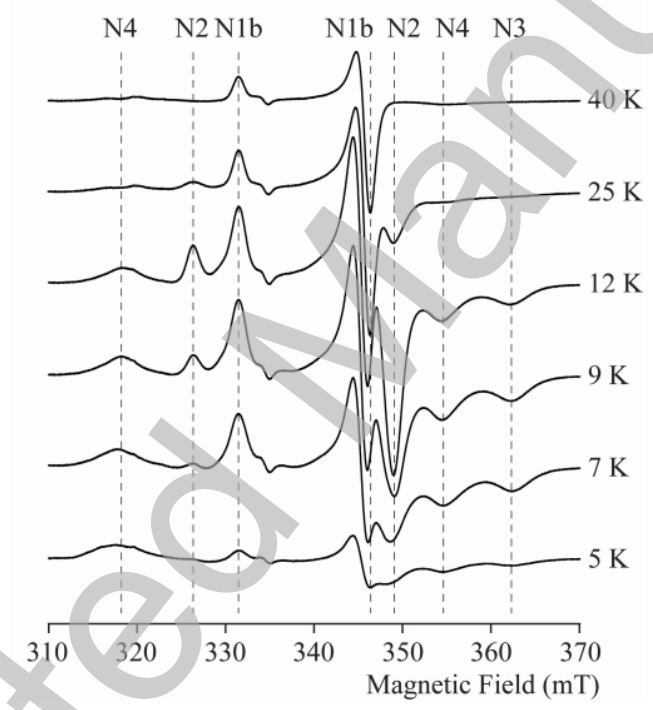




\section{Figure 4}

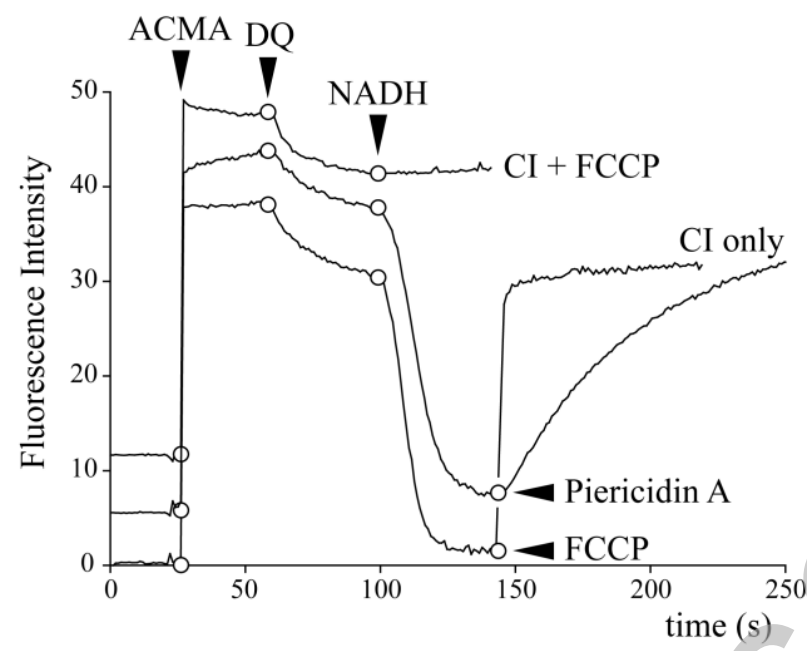

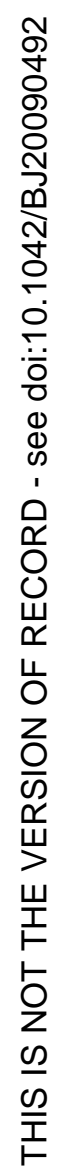




\section{Figure 5}

1. NUAM $(75 \mathrm{kDa})$

2. NUBM $(51 \mathrm{kDa})$

3. NUCM (49 kDa)

4. NUEM $(39 \mathrm{kDa})$

5. NUGM $(30 \mathrm{kDa})$

6. NUHM $(24 \mathrm{kDa})$

7 NUIM (TYKY)

8. NUJM (B14.7)

9. NUKM (PSST)

10. NUZM

11. NIMM (MWFE)

NB6M (B16.6)

12. N7BM (B17.2)

13. NUYM (18 kDa)

14. NIAM (ASHI)

15. NUMM (13 kDa)

16. NUFM (B13)

17. NIDM (PDSW)
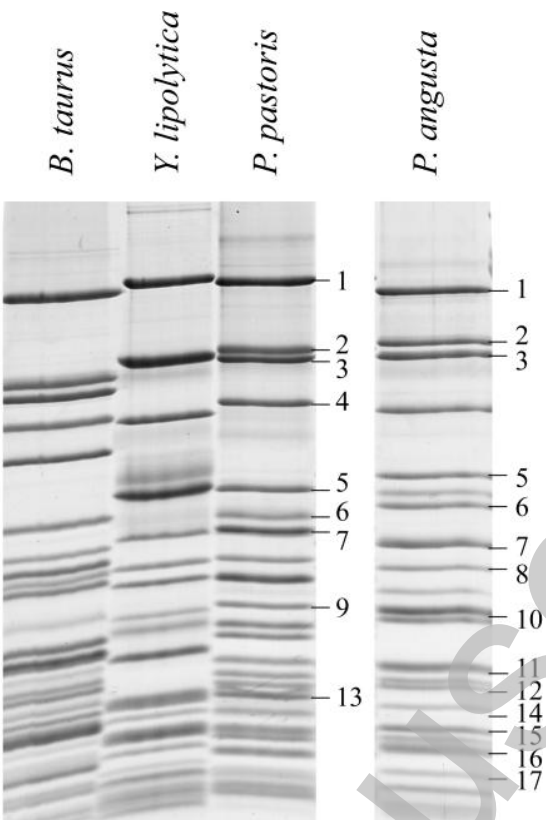

$-10$

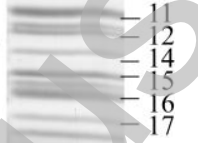

